\title{
Definitheitsbedingungen für relative Extrema bei Optimierungs- und Approximationsaufgaben
}

\author{
W. WETTERLING \\ Herrn Prof. Dr. Dr. h.c. L. Collatz zum 60 . Geburtstag gewidmet \\ Eingegangen am 6. Oktober 1969 \\ Summary. In this paper, sufficient and necessary maximum conditions are estab- \\ lished for a class of mathematical programming problems with an infinite set of \\ restrictions, which is described by a finite number of inequaiities. The criteria may \\ be applied to nonlinear approximation problems and to the numerical solution of
} boundary value problems.

\section{§1. Einleitung}

In diesem Beitrag werden hinreichende und notwendige Bedingungen für relative Extrema bei einem Typ zweistufiger Optimierungsaufgaben ( $\$ 3$ ) hergeleitet; das sind Aufgaben mit unendlich vielen Ungleichungen als Restriktionen, wobei die Menge der Restriktionen durch ein endliches System von Ungleichungen beschrieben wird. Die Betrachtung dieses Aufgabentyps ist durch die Anwendungsmöglichkeiten nahegelegt: Nichtlineare Probleme der Tschebyscheff-Approximation, insbesondere solche in mehreren reellen Variablen, werden hierdurch erfaßt $(\S 8)$, ferner Optimierungsprobleme, die bei der numerischen Behandlung von Randwertaufgaben nach dem Prinzip der lokal optimalen Schranken [6] auftreten. In diesen Fällen entspricht die Restriktionenmenge dem Bereich, in dem die Approximations- bzw. Randwertaufgabe formuliert ist. So ist diese Arbeit als Weiterführung des von und mit Collatz in [1] und [2], \$15 und weiterhin in $[6-8]$ verfolgten Programms der Anwendung von Approximations- und Optimierungsmethoden bei Randwertaufgaben anzusehen.

Bei den hier betrachteten Aufgaben werden über die Zielfunktion und die Restriktionsfunktionen keine generellen Konvexitätsvoraussetzungen gemacht; es ergeben sich schwache Definitheitsbedingungen für die Matrix der zweiten Ableitungen einer erweiterten Lagrange-Funktion.

Hinreichend für ein Extremum ist die Definitheit, notwendig die Semidefinitheit der quadratischen Form zu dieser Matrix im Tangentialraum an der betrachteten Stelle. Während dieser im Fall von endlich vielen Restriktionen ( $\$ 2$ ) durch ein homogenes lineares Gleichungssystem beschrieben wird, in dem die ersten Ableitungen der Restriktionsfunktionen auftreten, hat man im Fall der zweistufigen Aufgaben ( $\$ 3 \mathrm{ff}$.) auch lineare Relationen, die die zweiten Ableitungen enthalten; diese sind als linearisierte Enveloppenbedingungen zu deuten.

Die wichtige Frage nach numerischen Methoden zur Behandlung solcher Aufgaben wird hier nicht erörtert. Offenbar erfordern die verschiedenen speziellen 
Probleme, die sich der hier betrachteten allgemeinen Problemklasse unterordnen, verschiedenartige Algorithmen. Einige Hinweise findet man in [6] und [8]. Die in diesem Beitrag angegebenen Bedingungen bieten die Möglichkeit, das Ergebnis eines solchen Algorithmus, das im allgemeinen nur ein stationärer Wert ist, auf die Extremaleigenschaft zu prüfen.

\section{§ 2. Finite Maximumaufgaben}

Zunächst wird eine Aufgabe mit endlich vielen Restriktionen betrachtet; die hierüber bekannten Ergebnisse werden später benötigt:

Gesucht sind relative Maxima einer Funktion $F(x)$ auf einer Menge $M \subset R^{n}$, die durch

$$
M=\left\{x ; f_{j}(x) \leqq 0(j=1, \ldots, m)\right\}
$$

beschrieben ist. Dabei sind $F(x), f_{1}(x), \ldots, f_{m}(x)$ reellwertig definiert und zweimal stetig differenzierbar für alle $x \in R^{n}$. Mit $F^{\prime}(x)$ wird der Spaltenvektor $\operatorname{grad} F(x)$ mit den Komponenten $\partial F / \partial x_{i}(i=1, \ldots, n)$ bezeichnet, mit $F^{\prime \prime}(x)$ die Matrix der zweiten partiellen Ableitungen von $F$. Entsprechend sind $f_{j}^{\prime}(x)$ und $f_{j}^{\prime \prime}(x)$ zu verstehen.

Sei nun ein $\tilde{x} \in M$ gegeben; man möchte prüfen, ob $F$ an der Stelle $\tilde{x}$ ein relatives Maximum bezüglich $M$ hat. Hinreichend dafür sind folgende Bedingungen:

(a) Lokale Kuhn-Tucker-Bedingung. Für $j \in J \subset\{1, \ldots, m\}$, aber nicht notwendig nur für diese Indizes, ist $f_{j}(\tilde{x})=0$, und es gibt reelle Zahlen $u_{j}>0(j \in J)$ mit

$$
F^{\prime}(\tilde{x})-\sum_{i \in J} u_{j} f_{j}^{f}(\tilde{x})=0 .
$$

(b) Sei $H \subset R^{n}$ der lineare Teilraum der Vektoren $\xi \in R^{n}$ mit $\xi^{T} f_{j}^{\prime}(\tilde{x})=0 \quad(j \in J)$ $\left(\xi^{T}\right.$ ist der zum Spaltenvektor $\xi$ transponierte Zeilenvektor); die quadratische Form

ist negativ definit auf $H$.

$$
Q(\xi)=\xi^{T}\left[F^{\prime \prime}(\tilde{x})-\sum_{j \in J} u_{j} f_{j}^{\prime \prime}(\tilde{x})\right] \xi
$$

Sind die Bedingungen (a) und (b) erfüllt, so gibt es eine Umgebung von $\tilde{x}$, in deren Durchschnitt mit $M$ ( $\tilde{x}$ selbst ausgenommen) $F(x)<F(\tilde{x})$ ist.

Die entsprechenden notwendigen Bedingungen lauten: Sei $\tilde{x} \in M$ und $J$ nun die Menge aller Indizes $j$ mit $f_{j}(\tilde{x})=0$. Folgende constraint qualifications an der Stelle $\tilde{x}$ seien erfüllt:

Ist $\xi \in R^{n}$ ein Vektor mit $\xi^{T} f_{j}^{\prime}(\tilde{x}) \leqq 0(j \in J)$, so gibt es ein $t_{0}>0$ und eine für $0 \leqq t \leqq t_{0}$ stetig differenzierbare vektorwertige Funktion $x(t)$ mit $x(0)=\tilde{x}, d x(0) / d t=\xi$ und $x(t) \in M\left(0 \leqq t \leqq t_{0}\right)$. Ist $\xi^{x} f_{j}^{\prime}(\tilde{x})=0(j \in J)$, so gibt es ein solches $x(t)$, das uiberdies zweimal stetig differenzierbar ist und für das $f_{j}(x(t))=0\left(j \in J, 0 \leqq t \leqq t_{0}\right)$ ist.

Ist dann $F(x) \leqq F(\tilde{x})$ im Durchschnitt einer Umgebung von $\tilde{x}$ mit $M$, so gilt:

(a*) Es gibt $u_{j} \geqq 0(j \in J)$ mit (1).

(b*) $Q(\xi)$ ist negativ semidefinit auf $H$.

Man beachte jedoch, daB $J$ und damit $H$ und $Q$ jetzt anders definiert sind als im Fall der hinreichenden Bedingungen. 
Diese Aussagen sind in den Ergebnissen von McCormick [4] enthalten, der neben Ungleichungen auch Gleichungen als Restriktionen zuläßt.

Dort wird auBerdem gezeigt, daß die constraint qualifications sicher dann erfüllt sind, wenn die Vektoren $f_{j}^{\prime}(\tilde{x})(j \in J)$ linear unabhängig sind. Für das von McCormick mit indirektem Beweis hergeleitete, hinreichende Kriterium ist in [8] unter der Voraussetzung der Stetigkeit der dritten Ableitungen der auftretenden Funktionen ein direkter Beweis angegeben, bei dem eine Kugelumgebung von $\tilde{x}$ mit der genannten Eigenschaft konstruiert wird.

\section{§3. Zweistufige Maximumaufgaben, hinreichende Bedingungen}

Eine ähnliche Bedingung soll nun für folgenden Aufgabentyp, dessen Struktur die Bezeichnung zweistufig nahelegt, formuliert werden: Gesucht sind relative Maxima von $F(x)$ auf

dabei ist

$$
M=\{x ; f(x, y) \leqq 0(y \in Y)\} \subset R^{n} ;
$$

$$
Y=\left\{y ; g_{v}(y) \leqq 0(v=1, \ldots, N)\right\}<R^{m} .
$$

Die Funktionen $F, f, g_{v}$ seien reellwertig definiert und zweimal stetig differenzierbar im $R^{n}, R^{n} \times R^{m}$ bzw. $R^{m}$. Wie oben werden mit $F^{\prime}(x)$ und $f^{\prime}(x, y)$ die Vektoren der ersten Ableitungen nach den $x_{i}$ bezeichnet, ferner mit $f^{\prime}(x, y)$ und $g_{v}(y)$ die Vektoren der ersten Ableitungen nach den $y_{k}$, weiterhin mit $F^{\prime \prime}, f^{\prime \prime} f^{\prime \prime}, f^{\prime \prime}$ und $g_{v}$ die entsprechenden Matrizen der zweiten Ableitungen.

Wieder wird ein Punkt $\tilde{x} \in M$ betrachtet. Als hinreichend dafür, daß dort ein relatives Maximum von $F$ bezüglich $M$ vorliegt, erweisen sich die folgenden Bedingungen:

(A) Lokale Kuhn-Tucker-Bedingung. Für $y_{1}, y_{2}, \ldots, y_{p} \in Y$, aber nicht notwendig nur für diese Punkte, sei $f\left(\tilde{x}, y_{i}\right)=0$, und es gebe reelle Zahlen $u_{i}>0$ $(j=1, \ldots, p)$ mit

$$
F^{\prime}-\sum_{i=1}^{p} u_{j} f_{j}^{\prime}=0
$$

(das Argument $\tilde{x}$ wird hier und im folgenden häufig weggelassen, das Argument $y_{j}$ ebenfalls und durch einen Index $j$ angedeutet).

Als Funktion von $y$ auf der Menge $Y$ hat $f(\tilde{x}, y)$ bei $y=y_{j}$ Maxima mit dem Funktionswert $f\left(\tilde{x}, y_{j}\right)=0$. Diese Maxima sollen so beschaffen sein, daß ähnliche Definitheitsbedingungen wie in $\$ 2$ gelten.

(B) Für jedes $j(1 \leqq j \leqq p)$ gelte: Fïr $v \in M_{j} \subset\{1, \ldots, N\}$ und nur für diese Indizes ist $g_{v}\left(y_{j}\right)=0$, die Vektoren $g_{v i}=g_{\nu}^{*}\left(y_{j}\right)\left(\nu \in M_{j}\right)$ sind linear unabhängig, und es gibt reelle Zahlen $v_{v j} \geqq 0\left(\nu \in M_{j}\right)$ mit

Auf dem linearen Teilraum

$$
f_{i}-\sum_{v \in M_{j}} v_{p j} \dot{g}_{\nu j}^{*}=0
$$

$$
H_{j}=\left\{\eta ; \eta^{T} g_{v j}^{*}=0\left(\nu \in M_{j}\right)\right\}<R^{m}
$$


ist die quadratische Form

negativ definit.

$$
Q_{j}(\eta)=\eta^{T}\left(\ddot{f_{i}}-\sum_{v \in M_{i}} v_{\nu j} g_{v i}^{\ddot{*}}\right) \eta
$$

Bevor die eigentliche Definitheitsbedingung (C) formuliert werden kann, sind einige Zwischenüberlegungen nötig. Ist $m_{j}$ die Anzahl der Indizes in $M_{j}$, so hat $H_{j}$ die Dimension $m-m_{j}$.

Seien nun $\eta_{j}^{(1)}, \ldots, \eta_{j}^{\left(m-m_{j}\right)}$ Vektoren, die eine Basis von $H_{j}$ bilden. Hiermit wird das lineare Gleichungssystem

$$
\eta_{j}^{(k) T}\left[f_{j}^{\prime} \xi+\left(f_{i}-\sum_{\nu \in M_{j}} v_{v j} g_{v j}\right) \eta\right]=0 \quad\left(k=1, \ldots, m-m_{j}\right)
$$

aufgestellt. Es zeigt sich, daß es bei gegebenem $\xi \in R^{n}$ genau ein $\eta \in H_{j}$ als Lösung dieses Systems gibt. Der Ansatz

$$
\eta=\sum_{i=1}^{m-m_{j}} c_{i} \eta_{j}^{(i)}
$$

führt nämlich auf ein lineares Gleichungssystem für die Unbekannten $c_{i}$ mit der Koeffizientenmatrix

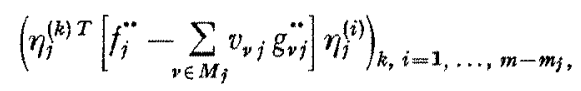

die wegen der Definitheit von $\left(f_{j}^{*}-\sum v_{y j} g_{v j}^{*}\right)$ nichtsingulär ist. So sind also einem $\xi \in R^{n}$ als Lösungen von (3) für $j=1, \ldots, p$ Vektoren $\eta_{1} \in H_{1}, \ldots, \eta_{p} \in H_{p}$ eindeutig zugeordnet, und zwar linear:

$$
\eta_{j}=P_{j} \xi \quad(j=1, \ldots, p)
$$

mit von $\xi$ unabhängigen $m \times n$-Matrizen $P_{j}$.

Die Definitheitsbedingung lautet dann

(C) Die quadratische Form

sei auf

$$
Q(\xi)=\xi^{T}\left[F^{\prime \prime}-\sum_{j=1}^{p} u_{j}\left(f_{j}^{\prime \prime}+2 P_{j}^{T} \tilde{f}_{j}^{\prime}+P_{j}^{T}\left(f_{j}^{*}-\sum_{v \in M_{j}} v_{v i} g_{v j}\right) P_{j}\right)\right] \xi
$$

negativ definit.

$$
H=\left\{\xi ; \xi^{T} f_{j}^{\prime}=0(j=1, \ldots, p)\right\}
$$

Diese Bedingung kann auch so formuliert werden: Es sei

$$
q\left(\xi, \eta_{j}\right)=\xi^{T}\left(F^{\prime \prime}-\sum u_{j} f_{j}^{\prime \prime}\right) \xi-2 \sum u_{j} \eta_{j}^{T} f_{j}^{\prime} \xi-\sum u_{j} \eta_{j}^{T}\left(f_{i}^{\prime \prime}-\sum v_{p j} g_{v j}^{*}\right) \eta_{j}<0
$$

für $\xi \in H, \eta_{j} \in H_{j}$, sofern $\xi$ und die $\eta_{j}$ durch (3) verknüpft sind und solange nicht $\xi=0$ und daher $\eta_{1}=\cdots=\eta_{p}=0$ ist. Wegen (3) ist hier

und daher

$$
\eta_{j}^{T} \ddot{f}_{j}^{\prime} \xi+\eta_{j}^{T}\left(f_{i}-\sum v_{v j} \dot{g}_{v i}^{*}\right) \eta_{j}=0
$$

$$
q(\xi, \eta)=\xi^{T}\left(F^{\prime \prime}-\sum u_{j} f_{j}^{\prime \prime}\right) \xi+\sum u_{j} \eta_{j}^{T}\left(\ddot{f_{j}}-\sum v_{v j} \ddot{g}_{v j}\right) \eta_{j} .
$$

Die negative Definitheit von $\xi^{T}\left(F^{\prime \prime}-\sum u_{j} f_{j}^{\prime \prime}\right) \xi$ (vgl. $\left.\S 2\right)$ ist also zusammen mit (B) hinreichend für (C), ist aber ersichtlich einschränkender als (C). 


\section{$\S 4$. Beweis der Maximaleigenschaft}

Satz. Sind fïr ein $\tilde{x} \in M$ die Bedingungen (A), (B), (C) erfiullt, so gibt es eine Kugel um $\tilde{x}$, in deren Durchschnitt mit $M$ ( $\tilde{x}$ selbst ausgenommen) $F(x)<F(\tilde{x})$ ist.

Beweis (indirekt). Aus der folgenden Annahme ist ein Widerspruch herzuleiten: Es gibt eine Folge $x_{1}, x_{2}, \ldots$ von Punkten $x_{k} \in M$ mit $x_{k} \neq \tilde{x}, \lim _{k \rightarrow \infty} x_{k}=\tilde{x}$ und

$$
F\left(x_{k}\right) \geqq F(\tilde{x}) .
$$

Sei $x_{k}-\tilde{x}=\delta_{k} \xi_{k}$ mit $\delta_{k}>0,\left\|\xi_{k}\right\|=1$ mit irgendeiner Vektornorm und daher $\lim \delta_{k}=0$. Die Folge der $\xi_{k}$ enthält eine konvergente Teilfolge. Ohne Beschränkung der Allgemeinheit sei also $\lim \xi_{k}=\xi$ mit $\|\xi\|=1$. Es ist

$$
\frac{1}{\delta_{k}}\left[F\left(\tilde{x}+\delta_{k} \xi_{k}\right)-F(\tilde{x})\right] \geqq 0
$$

und daher auch der Grenzwert $\xi^{T} F^{\prime}(\tilde{x}) \geqq 0$. Wegen $x_{k} \in M$ und $f\left(\tilde{x}, y_{j}\right)=0$ ist ferner

$$
\frac{1}{\delta_{k}}\left[f\left(\tilde{x}+\delta_{k} \xi_{k}, y_{j}\right)-f\left(\tilde{x}, y_{j}\right)\right] \leqq 0
$$

und daher $\xi^{T} f_{j}^{\prime} \leqq 0$. Mit (2) wird

$$
0 \leqq \xi^{T} F^{\prime}=\sum_{j=1}^{p} u_{j} \xi^{T} f_{i}^{\prime} \leqq 0 .
$$

Wegen $u_{j}>0$ ist $\xi^{T} F^{\prime}=\xi^{T} f_{1}^{\prime}=\cdots=\xi^{T} f_{b}^{\prime}=0$, also $\xi \in H$. Mit diesem $\xi$ sei $\eta_{i}=P_{j} \xi$ $(j=1, \ldots, p)$.

Für jeden Index $j(1 \leqq j \leqq p)$ gilt nun folgende Uberlegung: Es ist $g_{v}\left(y_{j}\right)=0$ und $\eta_{i}^{T} \dot{g}_{v}\left(y_{j}\right)=0\left(v \in M_{j}\right)$. In einem Intervall $0 \leqq \delta \leqq \Delta$ mit hinreichend kleinem positiven $\Delta$ gibt es einen Vektor $\psi_{j}=\psi_{j}(\delta) \in R^{m}$ mit $\psi_{j}^{T} \eta=0\left(\eta \in H_{j}\right)$ und

$$
g_{\nu}\left(y_{j}+\delta \eta_{j}+\psi_{j}(\delta)\right)=0 \quad\left(v \in M_{j}\right)
$$

der als Funktion von $\delta$ zweimal stetig differenzierbar und mit $\psi_{j}(0)=0$ eindeutig bestimmt ist. Um dies einzusehen, mache man den Ansatz

$$
\psi_{i}(\delta)=\sum_{\nu \in M_{i}} b_{\nu}(\delta) \dot{g_{\nu}}
$$

und wende bekannte Sätze über implizite Funktionen an (s. etwa [3], Kap. X). Die hier auftretende Jacobi-Matrix $\left(g_{v j}^{*} \dot{g}_{\mu j}^{*}\right)_{v, \mu \in M_{j}}$ ist wegen der linearen Unabhängigkeit der Vektoren $g_{v j}^{*}$ nichtsingulär. Aus (6) folgt weiterhin

$$
g_{v j} T\left(\eta_{j}+\frac{d \psi_{j}(0)}{d \delta}\right)=0 \quad\left(v \in M_{j}\right)
$$

wegen $g_{v j}^{*} \eta_{j}=0$ und der linearen Unabhängigkeit der $g_{v j}^{*}$ ist $d \psi_{j}(0) / d \delta=0$ und daher $\psi_{j}(\delta)=\delta^{2} \chi_{j}(\delta)$ mit gleichmäßig beschränktem $\chi_{j}(\delta)$ für $0 \leqq \delta \leqq \Delta$.

Sei $\Delta>0$ weiterhin so klein gewählt, daß $g_{v}\left(y_{j}+\delta \eta_{j}+\delta^{2} \chi_{j}(\delta)\right)<0$ ist für $\nu \notin M_{j}$ und $0 \leqq \delta \leqq \Delta$, also $y_{j}+\delta \eta_{j}+\delta^{2} \chi_{j}(\delta) \in Y$. 
Die Zahlen $\delta_{k}$ der obigen Folge liegen für hinreichend großes $k$ im Intervall $[0, \Delta]$. Für diese $k$ wird mit $\chi_{j k}=\chi_{j}\left(\delta_{k}\right)$

$$
\begin{aligned}
& F\left(\tilde{x}+\delta_{k} \xi_{k}\right)-\sum_{j=1}^{p} u_{j}\{\underbrace{f\left(\tilde{x}+\delta_{k} \xi_{k}, y_{j}+\delta_{k} \eta_{j}+\delta_{k}^{2} \chi_{j k}\right)}_{\leqq 0} \\
& -\sum_{v \in M_{j}} v_{v j} g_{=0} \underbrace{\left(y_{j}+\delta_{k} \eta_{j}+\delta_{k}^{2} \chi_{j k}\right)}_{=0}\} \\
& =F(\tilde{x})-\sum_{j=1}^{p} u_{j}\{\underbrace{f\left(\tilde{x}, y_{j}\right)}_{=0}-\sum_{\nu \in M_{j}} \underbrace{v_{v j} g_{\nu}\left(y_{j}\right)}_{=0}\} \\
& +\delta_{k}\left[\xi_{k}^{T}(\underbrace{F^{\prime}-\sum u_{j} f_{j}^{\prime}}_{=0})-\sum u_{j}\left(\eta_{j}+\delta_{k} \chi_{i k}\right)^{T}(\underbrace{f_{j}-\sum v_{v i} g_{v j}}_{=0})\right] \\
& +\frac{1}{2} \delta_{k}^{2} q\left(\xi_{k}, \eta_{j}\right)+o\left(\delta_{k}^{2}\right) .
\end{aligned}
$$

Für $k \rightarrow \infty$ hat man hiermit einen Widerspruch zwischen (4) und (5).

Bemerkung. Aus dem Beweis ist zu erkennen, daß man auch $\eta_{j}=\alpha_{j} P_{j} \xi$ mit beliebigen reellen $\alpha_{j}$ hätte wählen können, und zwar bereits in der Formulierung der Bedingung (C). Man erkennt aber, daB $\alpha_{j}=1$ als Maximalstelle von $\alpha_{j}\left(2-\alpha_{j}\right)$ ausgezeichnet ist und für $\alpha_{j} \neq 1$ zwar auch hinreichende, aber einschränkendere Bedingungen als für $\alpha_{j}=1$ herauskommen. Auch aus den folgenden notwendigen Bedingungen wird das deutlich.

\section{$\S 5$. Notwendige Maximalbedingungen}

Bei $\tilde{x}$ habe $F(x)$ bezüglich $M=\{x ; f(x, y) \leqq 0(y \in Y)\}$ ein schwaches relatives Maximum, d.h. im Durchschnitt einer Umgebung von $\tilde{x}$ mit $M$ sei $F(x) \leqq F(\tilde{x})$. Sei dabei $f(\tilde{x}, y)=0$ genau für $y=y_{1}, \ldots, y_{p} \in Y$ (es sind also nur endlich viele Nullstellen von $f(\tilde{x}, y)$ in $Y$ zugelassen). Außer dieser Einschränkung werden noch einige weitere Bedingungen (constraint qualifications) gefordert, zunächst:

(I) Ist $\xi \in R^{n}$ ein Vektor mit $\xi^{T} f^{\prime}\left(\tilde{x}, y_{j}\right) \leqq 0(j=1, \ldots, p)$, so gibt es ein $t_{0}>0$ und eine fiir $0 \leqq t \leqq t_{0}$ stetig differenzierbare vektorwertige Funktion $x(t)$ mit $x(0)=\tilde{x}$, $d x(0) / d t=\xi$ und $x(t) \in M\left(0 \leqq t \leqq t_{0}\right)$.

Für ein beliebiges solches $\xi$ ist dann

$$
\xi^{T} F^{\prime}(\tilde{x})=\frac{d}{d t} F(x(t))_{(t=0)} \leqq 0,
$$

es gibt also kein $\xi \in R^{n}$ mit $\xi^{T} f^{\prime}\left(\tilde{x}, y_{j}\right) \leqq 0(j=1, \ldots, p)$ und $\xi^{T} F^{\prime}(\tilde{x})>0$.

Nach dem Alternativsatz für Systeme von linearen Ungleichungen (Lemma von Farkas, [2], §5) gibt es $u_{j} \geqq 0(j=1, \ldots, p)$ mit

$$
F^{\prime}(\tilde{x})-\sum_{j=1}^{p} u_{j} f^{\prime}\left(\tilde{x}, y_{j}\right)=0
$$

Weiterhin sei folgende Bedingung erfüllt: 
(II) Ist $\xi \in R^{n}$ ein Vektor mit $\xi^{T} f^{\prime}\left(\tilde{x}, y_{j}\right)=0(j=1, \ldots, p)$, so gibt es fir $0 \leqq t \leqq t_{1}$ $\left(t_{1}>0\right)$ ein zweimal stetig differenzierbares $x(t) \in M$ mit $x(0)=\tilde{x}, d x(0) / d t=\xi$ und zugeordnete, ebenfalls zweimal stetig differenzierbare $z_{j}(t) \in Y$ mit $z_{j}(0)=y_{j}$ und

$$
f\left(x(t), z_{j}(t)\right)=0 \quad\left(0 \leqq t \leqq t_{1} ; j=1, \ldots, p\right) .
$$

Seien schlieBlich die Maximalstellen $y_{j}$ von $f(\tilde{x}, y)$ wie folgt beschaffen:

(III) Für $j=1, \ldots, p$ gilt: Es is $g_{v}\left(y_{j}\right)=0$ genau für $v \in M_{j}<\{1, \ldots, N\}$, die Vektoren $g_{v j}^{\cdot}\left(v \in M_{j}\right)$ sind linear wnabhängig, es ist

$$
\dot{f_{j}}-\sum_{v \in M_{j}} v_{v i} \dot{g}_{v j}^{*}=0
$$
mit $v_{v j}>0\left(v \in M_{j}\right)$, und die Matrix $f_{j}^{*}-\sum_{\nu \in M_{i}} v_{v j} \ddot{g}_{v j}$ ist negativ definit auf $H_{j}=$
$\left\{\eta ; \eta^{T} g_{v j}^{*}=0\left(\nu \in M_{j}\right)\right\}$.

Sei nun $\xi \in H=\left\{\xi ; \xi^{T} f_{j}^{\prime}=0(j=1, \ldots, p)\right\}$ und hierzu $x(t)$ und $z_{1}(t), \ldots, z_{p}(t)$ gemäß (II) gegeben. Setzt man $d z_{j}(0) / d t=\eta_{j}$, so wird nach $(8) \xi^{T} f_{j}^{\prime}+\eta_{j}^{T} f_{j}=0$, also $\eta_{j}^{T} f_{j}=0(j=1, \ldots, p)$. Wegen $z_{j}(t) \in Y$ ist $\eta_{j}^{T} g_{v j} \leqq 0$ und wegen $v_{v j}>0$ und (9) sogar

$$
\eta_{j}^{T} g_{v j}^{*}=0 \quad\left(\nu \in M_{j} ; j=1, \ldots, p\right) .
$$

Für festes $t \in\left[0, t_{1}\right]$ sind die Punkte $y=z_{j}(t)$ Maximalstellen von $f(x(t), y)$ bezüglich $Y$. Es zeigt sich, daß wenigstens in einem Teilintervall von $\left[0, t_{1}\right]$ die notwendigen Bedingungen von McCormick (\$2) gelten. Wegen der Stetigkeit der $g_{v}$ ist nämlich für $0 \leqq t \leqq t_{2}$ mit $0<t_{2} \leqq t_{1} g_{\nu}\left(z_{j}(t)\right)<0\left(v \notin M_{j}\right)$. Sei $M_{j}(t)<M_{j}$ die Menge der Indizes $v$, für die $g_{v}\left(z_{j}(t)\right)=0$ ist $\left(0 \leqq t \leqq t_{2}\right)$. Auch die Vektoren $g_{v}^{*}\left(z_{j}(t)\right)$ hängen stetig von $t \mathrm{ab}$, und darum gibt es ein $t_{3}$ mit $0<t_{3} \leqq t_{2}$, so daß die $g_{v}\left(z_{j}(t)\right)\left(\nu \in M_{j}(t)\right)$ linear unabhängig sind, sofern $t \in\left[0, t_{3}\right]$ ist. Nach $\S 2$ gibt es Zahlen $w_{\nu j}(t) \geqq 0\left(\nu \in M_{j}(t)\right)$ mit

$$
f\left(x(t), z_{j}(t)\right)-\sum_{v \in M_{j}(t)} w_{v i}(t) g_{p}^{\cdot}\left(z_{j}(t)\right)=0
$$

Betrachtet man (9) und (11), so erkennt man: Für $t=0$ liegt der Vektor $f^{*}(x(t)$, $\left.z_{j}(t)\right)$ im relativen Inneren des von den Vektoren $g_{v}\left(z_{j}(t)\right)$ aufgespannten Kegels. Alle diese Vektoren hängen stetig von $t \mathrm{ab}$. Darum gibt es ein $t_{4}$ mit $0<t_{4} \leqq t_{3}$, so daB für $t \in\left[0, t_{4}\right]$ die Gl. (11) nur dann gelten kann, wenn $M_{j}(t)=M_{j}(0)=M_{i}$ und $w_{y}(t)>0$ ist. Es ist also auch $g_{y}\left(z_{j}(t)\right)=0$ in $\left[0, t_{4}\right]$ für alle $v \in M_{j}$. Ebenso wie die Vektoren $f^{*}$ und $g_{v}^{*}$ im Gleichungssystem (11) hängt auch dessen Lösung $w_{v j}(t)$ mit dem Anfangswert $w_{v j}(0)=v_{v j}$ im Intervall $\left[0, t_{4}\right]$ stetig differenzierbar von $t$ ab. Aus (11) folgt dann

$$
\ddot{f}_{j}^{\prime} \xi+\left(\ddot{f_{j}}-\sum_{v \in M_{j}} v_{v j} \dot{g}_{v j}\right) \eta_{i}-\sum_{v \in M_{j}} \frac{d w_{v j}(0)}{d t} \dot{g}_{v j}=0
$$

Ist $\left\{\eta_{j}^{(\mathbf{l})}, \ldots, \eta_{i}^{\left(m-m_{j}\right)}\right\}$ eine Basis von $H_{j}=\left\{\eta ; \eta^{T} g_{v j}^{\cdot}=0\left(\nu \in M_{j}\right)\right\}$, so wird

$$
\eta_{i}^{(k)}\left[f_{j}^{\prime \prime} \xi+\left(f_{i}-\sum_{\nu \in M_{j}} v_{v_{j}} g_{v j}\right) \eta_{j}\right]=0 \quad\left(k=1, \ldots, m-m_{j}\right) .
$$


Als zugeordnete $z_{j}(t)$ in (II) kommen also nur solche in Frage, für die mit $\eta_{j}=d z_{j}(0) / d t$ das lineare Gleichungssystem (12) erfüllt ist. Weil $x(t) \in M$ für $0 \leqq t \leqq t_{4}, F$ bei $\tilde{x}=x(0)$ maximal und

ist, wird

$$
\frac{d}{d t} F(x(t))_{(t=0)}=\xi^{T} F^{\prime}=\sum_{j=1}^{p} u_{j} \xi^{T} f_{j}^{\prime}=0
$$

$$
\frac{d^{2}}{d t^{2}} F(x(t))_{(t=0)}=\alpha^{T} F^{\prime}+\xi^{T} F^{\prime \prime} \xi \leqq 0,
$$

wobei $\alpha=d^{2} x(0) / d t^{2}$ ist. Weiterhin ist mit $\beta_{j}=d^{2} z_{j}(0) / d t^{2}$

$\frac{d^{2}}{d t^{2}} f\left(x(t), z_{j}(t)\right)_{(t=\mathbf{0})}=\alpha^{T} f_{j}^{\prime}+\beta_{j}^{T} \dot{f}_{j}+\xi^{T} f_{j}^{\prime \prime} \xi+2 \xi^{T} f_{j}^{\prime} \eta_{j}+\eta_{j}^{T} f_{j} \eta_{j}=0 \quad(j=1, \ldots, p)$

und

$$
\frac{d^{2}}{d t^{2}} g_{v}\left(z_{j}(t)\right)_{(t=0)}=\beta_{j}^{T} \dot{g_{v j}}+\eta_{j}^{T} g_{v j} \eta_{j}=0 \quad\left(v \in M_{j} ; j=1, \ldots, p\right)
$$

Mit (7) und (9) wird

$$
\begin{aligned}
\frac{a^{2}}{d t^{2}} F(x(t))_{(t=0)}= & \xi^{T}\left(F^{\prime \prime}-\sum_{j=1}^{p} u_{j} f_{j}^{\prime \prime}\right) \xi-2 \sum_{j=1}^{p} u_{j} \xi^{T} f_{j}^{\prime} \eta_{j} \\
& -\sum_{j=1}^{p} u_{j} \eta_{i}^{T}\left(\ddot{f_{j}}-\sum_{\nu \in M_{j}} v_{\nu} g_{\nu j}\right) \eta_{j} \leqq 0
\end{aligned}
$$

und zwar dies für alle $\xi \in H$ und die ihnen durch (12) eindeutig zugeordneten $\eta_{j} \in H_{j}$.

Satz. Sei $F(x) \leqq F(\tilde{x})$ im Durchschnitt einer Umgebung von $\tilde{x}$ mit $M$, und sei $f(\tilde{x}, y)=0$ genau fiur $y=y_{1}, \ldots, y_{p} \in Y$. Ist dann die Bedingung (I) erfüllt, so gilt die lokale Kuhn-Tucker-Bedingung (7). Sind außerdem die Bedingungen (II) und (III) erfïllt, so gilt die Semidefinitheitsbedingung (13).

\section{§6. Die constraint qualifications}

Die Bedingungen (I) und (II) sind nicht so einschränkend, wie es zunächst scheint. Sie besagen, daß man an einer Maximalstelle nur dann die angegebenen notwendigen Bedingungen folgern kann, wenn diese Stelle nicht ein Randpunkt von $M$ von dem Typ der aus der Theorie der linearen Optimierung bekannten entarteten Ecken ist. Hier gilt ein ähnlicher Satz wie im Fall der finiten Maximumaufgaben $(\$ 2)$.

Satz. Sei die Menge $Y$ beschränkt, seien ferner $f(x, y), g_{1}(y), \ldots, g_{N}(y)$ dreimal stetig differenzierbar und $y_{1}, \ldots, y_{p}$ alle Punkte in $Y$ mit $f(\tilde{x}, y)=0$. Ist dann die Bedingung (III) erfiullt und sind die Vektoren $f^{\prime}\left(\tilde{x}, y_{j}\right)(j=1, \ldots, p)$ linear unabhängig, so sind auch (I) und (II) erfüllt.

Beweis. Sei $\xi \in R^{n}$ ein Vektor mit $\xi^{T} f^{\prime}\left(\tilde{x}, y_{j}\right) \leqq 0(j=1, \ldots, p)$, und zwar sei

$$
\xi^{T} f^{\prime}\left(\tilde{x}, y_{j}\right) \begin{cases}=0 & (j=1, \ldots, r) \\ <0 & (j=r+1, \ldots, p) .\end{cases}
$$


Zum Nachweis von (II) ist hier $r=p$ anzunehmen, während (I) für geeignetes $r$ $(0 \leqq r \leqq p)$ folgt, wenn man ohne Beschränkung der Allgemeinheit annimmt, daß die Numerierung der $y_{j}$ passend gewählt ist.

Es werden vektorwertige Funktionen $x(t), z_{1}(t), \ldots, z_{r}(t)$ konstruiert, die in einem Intervall $\left[0, t_{1}\right]$ mit $t_{\mathbf{1}}>0$ zweimal stetig differenzierbar sind und für die

$$
\begin{aligned}
& \left.\begin{array}{l}
x(0)=\tilde{x}, \quad d x(0) / d t=\xi, \\
z_{j}(0)=y_{j} \quad(j=1, \ldots, r), \\
f\left(x(t), z_{j}(t)\right)=0 \\
g_{\nu}\left(z_{j}(t)\right)=0 \quad\left(\nu \in M_{j}\right)
\end{array}\right\} \quad\left(j=1, \ldots, r ; 0 \leqq t \leqq t_{1}\right)
\end{aligned}
$$

gilt. Bei $y=z_{j}(t)$ soll eine Maximalstelle von $f(x(t), y)$ bezüglich $Y$ liegen. Dort werden daher noch die lokalen Kuhn-Tucker-Bedingungen gefordert:

$$
f^{\prime}\left(x(t), z_{j}(t)\right)-\sum_{\nu \in M_{f}} w_{v j}(t) g_{v}^{\cdot}\left(z_{j}(t)\right)=0 \quad\left(j=1, \ldots, r ; 0 \leqq t \leqq t_{1}\right)
$$

mit $w_{v j}(0)=v_{v}$. Wenn wie oben $m_{j}$ die Anzahl der Indizes in $M_{j}$ ist, hat man mit (14), (15) und (16)

$$
r+\sum_{j=1}^{p} m_{j}+m r
$$

Gleichungen. Für $x(t)$ wird der Ansatz

$$
x(t)=\tilde{x}+t \xi+\sum_{k=1}^{r} c_{k}(t) f^{\prime}\left(\tilde{x}, y_{k}\right)
$$

gemacht.

Man hat dann $r$ reellwertige Funktionen $c_{k}(t), \sum m_{j}$ reellwertige Funktionen $w_{v j}(t)$ und $m r$ Komponenten der vektorwertigen Funktionen $z_{j}(t)$ zu den Anfangswerten

$$
c_{k}(0)=0, \quad w_{v j}(0)=v_{v j}, \quad z_{j}(0)=y_{j}
$$

aus den Gln. (14), (15) und (16) zu bestimmen. Für die Anfangswerte sind diese Gleichungen erfüllt. Die schon in $\$ 4$ benutzten Sätze über implizite Funktionen sind anwendbar, denn die Jacobi-Matrix an der Stelle $t=0$ ist nichtsingulär. Sie ist von der Form

$$
\left.\left(\begin{array}{ccc}
A & 0 & B \\
0 & 0 & C \\
D & \underbrace{-C^{T}}_{r} & \underbrace{G}_{m r}
\end{array}\right)\right\} \begin{aligned}
& r \\
& \sum m_{j}
\end{aligned} m_{j}
$$

Dabei ist $A=\left(f_{j}^{\prime} f_{k}^{\prime}\right)_{j, k=1, \ldots, r}$ wegen der linearen Unabhängigkeit der $f_{j}^{\prime}$ nichtsingulär. Weiterhin ist

$$
B=\left(\begin{array}{ccc}
f_{1}^{*} & 0 & \ldots \\
0 & f_{2}^{T} & \ldots \\
. & \cdot & .
\end{array}\right),
$$


$C$ ist entsprechend aus den Vektoren $g_{y j}^{\cdot T}$ aufgebaut, $D$ aus Vektoren $f_{i}^{\prime} f_{k}^{\prime}$, und $G$ ist eine Blockmatrix mit $r m \times m$-Matrizen $f_{i}-\sum v_{v j} g_{v i}^{*}$ längs der Hauptdiagonale, sonst mit Nullen ausgefüllt. Die Matrix (18) ist nichtsingulär, wenn es die Matrix

$$
\left(\begin{array}{ccc}
A & 0 & 0 \\
0 & 0 & C \\
D & -C^{T} & G
\end{array}\right)
$$

ist, denn diese entsteht wegen (9) aus (18), wenn man jeweils das $v_{v}$-fache der Zeilen von $\left(\begin{array}{llll}0 & 0 & C\end{array}\right)$ von den entsprechenden Zeilen $\left(\begin{array}{lll}A & 0 & B\end{array}\right)$ subtrahiert. Es bleibt zu zeigen, daB

$$
\left(\begin{array}{cc}
0 & C \\
-C^{T} & G
\end{array}\right)
$$

nichtsingulär ist. Es genügt, dies für eine Matrix

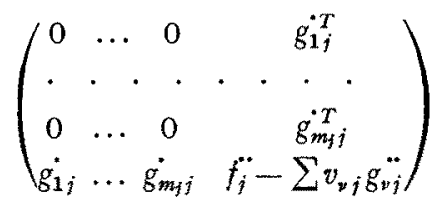

unter der Annahme $M_{j}=\left\{1, \ldots, m_{j}\right\}$ zu zeigen. Wäre sie singulär, so gäbe es Zahlen $a_{1}, \ldots, a_{m}$, und einen Vektor $\gamma \in R^{m}$, nicht sämtlich $=0$, mit

$$
\begin{gathered}
\sum a_{v} \dot{g_{v i}}+\left(\ddot{f_{j}}-\sum v_{v j} \ddot{g_{v j}}\right) \gamma=0, \\
g_{v j}^{* T} \gamma=0 \quad\left(\nu \in M_{j}\right) .
\end{gathered}
$$

Die $g_{v j}^{\cdot}$ sind in (III) als linear unabhängig vorausgesetzt, darum ist $\gamma \neq 0$. Multipliziert man (19) von links mit $\gamma^{T}$, so wird $\gamma^{T}\left(\ddot{f}-\sum v_{v j} \ddot{g_{i j}}\right) \gamma=0$ mit $\gamma \neq 0$ im Widerspruch zur Definitheitsvoraussetzung in (III). Die Sätze über implizite Funktionen besagen dann, daß es ein $t_{1}>0$ und im Intervall $\left[0, t_{1}\right]$ zweimal stetig differenzierbare $c_{k}(t), z_{j}(t), w_{v j}(t)$ gibt, die mit $c_{k}(0)=0, z_{j}(0)=y_{j}, w_{v j}(0)=v_{v j}$ eindeutig bestimmt sind. Da (16) erste Ableitungen enthält, mußte im Satz die Stetigkeit der dritten Ableitungen von $f$ und $g_{v}$ vorausgesetzt werden.

Es ist noch $d x(0) / d t=\xi$ zu zeigen. Aus (14) folgt

$$
f_{j}^{\prime T}\left(\xi+\sum_{k=1}^{r} \frac{d c_{k}(0)}{d t} f_{k}^{\prime}\right)+f_{j}^{T} \frac{d z_{j}(0)}{d t}=0 \quad(j=1, \ldots, r) .
$$

Hier ist $f_{j}^{T} \xi=0$ und auch $f_{j}^{T} d z_{j}(0) / d t=0$ wegen (9) und $g_{v j}^{\top} d z_{j}(0) / d t=0$, was aus (15) folgt. Da die Matrix $\left(f_{j}^{T} f_{k}^{\prime}\right)_{j, k=1, \ldots, r}$ nichtsingulär ist, wird $d c_{k}(0) / d t=0$ $(k=1, \ldots, r)$ und damit $d x(0) / d t=\xi$.

Für $v \notin M_{j}$ ist $g_{\nu}\left(y_{j}\right)<0$ und darum $g_{\nu}\left(z_{j}(t)\right)<0$ für $0 \leqq t \leqq t_{2}$ mit $0<t_{2} \leqq t_{1}$, also $z_{j}(t) \in Y$ für $t \in\left[0, t_{2}\right], j=1, \ldots, r$.

Schließlich ist zu zeigen, daß $x(t) \in M$, also $f(x(t), y) \leqq 0(y \in Y)$ ist in einem Intervall $\left[0, t^{*}\right]$ mit $t^{*}>0$. Hierzu werden Kugelumgebungen von $y_{1}, \ldots, y$, und von $y_{r+1}, \ldots, y_{p}$ konstruiert, und es ist zu unterscheiden, ob $y$ in einer solchen Umgebung liegt oder nicht. 
Sei zunächst $1 \leqq j \leqq r$. Für hinreichend kleine $t>0$ ist $y=z_{j}(t)$ Maximalstelle von $f(x(t), y)$ bezüglich $Y$ zum Maximalwert $f\left(x(t), z_{j}(t)\right)=0$. Nach (16) und wegen $w_{v j}(0)=v_{v j}>0\left(v \in M_{j}\right)$ ist nämlich für kleine positive $t$ die lokale KuhnTucker-Bedingung (a) von $\$ 2$ erfüllt. Die für $t=0$ durch (III) vorausgesetzte Definitheitsbedingung (b) ist ebenfalls aus Stetigkeitsgründen für kleine positive $t$ erfüllt. Damit gibt es für $t \in\left[0, t_{3}\right]$ mit $0<t_{3} \leqq t_{2}$ eine Umgebung von $z_{j}(t)$, in deren Durchschnitt mit $Y\left(z_{i}(t)\right.$ selbst ausgenommen)

$$
f(x(t), y)<f\left(x(t), z_{j}(t)\right)=0
$$

ist. Man kann hierfür die in [8] konstruierten Kugelumgebungen wählen, und zwar so, daB deren Radius stetig von $t$ abhängt (hier wird noch einmal die Stetigkeit der dritten Ableitungen von $f$ und $g_{v}$ benutzt). Sei also $\varrho_{j}(t)$ der Radius der Kugelumgebung um $z_{j}(t)$, wobei $\varrho_{j}(t)>0$ und stetig ist in einem Intervall $\left[0, t_{4}\right]$ mit $t_{4}>0$, und zwar dies für alle $j$ mit $1 \leqq j \leqq r$. Nun wird $t_{5}$ mit $0<t_{5} \leqq t_{\dot{4}}$ so klein gewählt, daß

$$
\max _{\left[0, t_{b}\right]}\left\|y_{j}-z_{j}(t)\right\| \leq \frac{1}{3} \min _{\left[0, t_{b}\right]} \varrho_{j}(t)
$$

Ist für $j=1, \ldots, r$. Dabei ist $\|\cdot\|$ die euklidische Vektornorm im $R^{m}$. Die Kugel $K_{j}$ mit dem Radius $\tau_{j}=\frac{1}{2} \min _{\left[0, t_{\mathrm{b}}\right]} \varrho_{j}(t)$ um $y_{j}$ enthält dann $y_{j}$ und $z_{j}(t)\left(0 \leqq t \leqq t_{5}\right)$ in ihrem Inneren. Andererseits ist $K_{j}$ enthalten in den Kugeln um $z_{j}(t)$ mit den Radien $\varrho_{j}(t)$ für alle $t \in\left[0, t_{5}\right]$, denn aus $\left\|y-y_{j}\right\| \leqq \tau_{j}$ folgt

$$
\left\|y-z_{j}(t)\right\| \leqq \tau_{j}+\left\|y_{j}-z_{j}(t)\right\| \leqq \frac{5}{6} \varrho_{j}(t) .
$$

Für $y \in Y \cap\left(K_{1} \cup \cdots \cup K_{r}\right)$ ist damit nach $(20) f(x(t), y) \leqq 0\left(0 \leqq t \leqq t_{5}\right)$.

Nun sei $r+1 \leqq j \leqq p$. Es ist $f\left(\tilde{x}, y_{j}\right)=0$ und $f(\tilde{x}, y)<0$ für $0<\left\|y-y_{j}\right\| \leqq s$, $y \in Y$ mit hinreichend kleinem $s>0$. Wegen $\xi^{T} f^{\prime}\left(\tilde{x}, y_{j}\right)<0$ kann $s>0$ so klein gewählt werden, daß $\xi^{T} f^{\prime}(\tilde{x}, y)<0$ für $\left\|y-y_{j}\right\| \leqq s$ wird. Im Durchschnitt von $Y$ mit der Kugel $K_{j}$ um $y_{j}$ mit dem Radius $s$ ist dann $f(x(t), y)<0$ (abgesehen von $\left.f\left(\tilde{x}, y_{j}\right)=0\right)$ für $t \in\left[0, t_{6}\right]$ mit $t_{6}>0$.

Sei schließlich $y \in L=Y-\bigcup_{j=1}^{p} K_{j}$; dort ist $f(\tilde{x}, y) \leqq \sigma<0$, denn $y_{1}, \ldots, y_{p}$ sind die einzigen Nullstellen von $f(\tilde{x}, y)$ in $Y$ und $Y$ ist beschränkt und abgeschlossen. Darum gibt es ein $t_{7}>0$, so daß $f(x(t), y) \leqq 0$ ist für $y \in L$ und $0 \leqq t \leqq t_{7}$. Insgesamt ist, wenn man $t^{*}=\min \left(t_{5}, t_{6}, t_{7}\right)$ setzt,

$$
f(x(t), y) \leqq 0 \quad\left(y \in Y, 0 \leqq t \leqq t^{*}\right)
$$

\section{$\S 7$. Beispiel}

Im Beispiel ist $n=m=2$ und $N=1$ bzw. $=2$.

$$
\begin{aligned}
F(x) & =x_{2}+c x_{1}^{2}, \\
f(x, y) & =\left(x_{1}-y_{1}\right)^{2}+\left(x_{2}-y_{2}\right)^{2}-4 \quad(\leqq 0), \\
g_{1}(y) & =y_{1}^{2}+y_{2}^{2}-1 \quad(\leqq 0) .
\end{aligned}
$$


Hiermit wird $M=\left\{x ; x_{1}^{2}+x_{2}^{2} \leqq 1\right\}$. Als Maximalstelle kommt $\tilde{x}=\left(\begin{array}{l}0 \\ 1\end{array}\right)$ in Betracht. Dann wird $f\left(\tilde{x}, y_{1}\right)=0$ bei $y_{1}=\left(\begin{array}{r}0 \\ -1\end{array}\right)$. Weiterhin ist

Es ist

$$
\begin{gathered}
F^{\prime}(\tilde{x})=\left(\begin{array}{l}
0 \\
1
\end{array}\right), \quad f^{\prime}\left(\tilde{x}, y_{1}\right)=\left(\begin{array}{l}
0 \\
4
\end{array}\right), \quad u_{1}=\frac{1}{4}, \\
f^{\prime}\left(\tilde{x}, y_{1}\right)=\left(\begin{array}{r}
0 \\
-4
\end{array}\right), \quad g_{1}\left(y_{1}\right)=\left(\begin{array}{r}
0 \\
-2
\end{array}\right), \quad v_{11}=2 .
\end{gathered}
$$

$$
q\left(\xi, \eta_{1}\right)=2 c \xi_{1}^{2}-\frac{1}{2}\left(\xi_{1}^{2}+\xi_{2}^{2}\right)+\left(\xi_{1} \eta_{1}+\xi_{2} \eta_{2}\right)+\frac{1}{2}\left(\eta_{1}^{2}+\eta_{2}^{2}\right) .
$$

$\xi \in H$ besagt $\xi_{2}=0, \eta \in H_{1}$ besagt $\eta_{2}=0$. (3) besagt schließlich $\xi_{1}+\eta_{1}=0$. Damit wird $q=(2 c-1) \xi_{1}^{2}$. Dies ist negativ definit für $c<\frac{1}{2}$, semidefinit für $c \leqq \frac{1}{2}$. Tatsächlich hat $F$ im Falle $c \leqq \frac{1}{2}$ bei $\tilde{x}$ ein Maximum bezüglich $M$, für $c>\frac{1}{2}$ jedoch nicht.

Nun werde $g_{2}(y)=y_{1} \leqq 0$ als $Y$ beschreibende Ungleichung hinzugenommen. Dann ist

$$
M=\left\{x ;-\sqrt{3-x_{2}^{2}-2\left|x_{2}\right|} \leqq x_{1} \leqq \sqrt{1-x_{2}^{2}}\right\} .
$$

Es wird $g_{2}=\left(\begin{array}{l}1 \\ 0\end{array}\right)$ und damit $v_{12}=0 . \eta \in H_{1}$ besagt nun $\eta_{1}=\eta_{2}=0$, wegen $H_{1}=\{0\}$ hat man keine Bedingungen (3), und es wird $q=\left(2 c-\frac{1}{2}\right) \xi_{1}^{2}$. Negative Definitheit hat man für $c<\frac{1}{4}$, Semidefinitheit für $c \leqq \frac{1}{4}$ und ein Maximum bei $\tilde{x}$ für $c \leqq \frac{1}{4}$. Obwohl die Bedingung (III) wegen $v_{12}=0$ nicht gilt, ist die notwendige Maximalbedingung genau für die Parameterwerte $c$ erfüllt, für die bei $\tilde{x}$ ein Maximum liegt.

\section{§ 8. Approximationsaufgaben}

Die folgende Aufgabe der Tschebyscheff-Approximation kann als Problem des in $\$ 3$ behandelten Typs geschrieben werden: Unter den Nebenbedingungen

$$
|\varphi(y)-\Phi(y, z)| \leqq \varepsilon \quad\left(y \in \widehat{Y} \subset R^{m}\right)
$$

soll $\varepsilon$ möglichst klein gemacht werden durch passende Wahl von $z \in R^{n-1}$. Dabei sei $\hat{Y}$ durch endlich viele Ungleichungen $g_{\nu}(z) \leqq 0(\nu=1, \ldots, N)$ beschrieben. Alle auftretenden Funktionen seien zweimal stetig differenzierbar im ganzen Raum.

Wenn man (21) als

$$
\left.\begin{array}{r}
\varphi(y)-\Phi(y, z)-\varepsilon \leqq 0 \\
-\varphi(y)+\Phi(y, z)-\varepsilon \leqq 0
\end{array}\right\}(y \in \widehat{Y})
$$

schreibt, $x=\left(z_{1}, \ldots, z_{n-1}, \varepsilon\right)^{T}$ setzt und als $Y$ zwei Exemplare von $\hat{Y}$ wählt, hat man eine Aufgabe wie in $\S 3$. Hier sollen die hinreichenden Bedingungen des $\S 3$ für ein relatives Minimum $\tilde{\varepsilon}$ von $\varepsilon$ an einer Stelle $z=\tilde{z}$ formuliert werden. Die Bezeichnungen entsprechen den bisher verwendeten, jedoch ist $\Phi^{\prime}=\left(\partial \Phi / \partial z_{1}\right.$, $\left.\ldots, \partial \Phi / \partial z_{n-1}\right)^{T}$.

(A') Für $y_{1}, \ldots, y_{r} \in \hat{Y}$ sei $\varphi\left(y_{j}\right)-\Phi\left(y_{j}, \tilde{z}\right)=\tilde{\varepsilon}$ und für $y_{r+1}, \ldots, y_{p} \in \hat{Y}$ sei

$$
\varphi\left(y_{j}\right)-\Phi\left(y_{j}, \tilde{z}\right)=-\tilde{\varepsilon} \text {. }
$$


Im übrigen sei

$$
|\varphi(y)-\Phi(y, \tilde{z})| \leqq \varepsilon \quad(y \in \widehat{Y}) .
$$

Es gebe reelle Zahlen $u_{j}>0(j=1, \ldots, r), u_{j}<0(j=r+1, \ldots, p)$ mit

$$
\sum_{j=1}^{p} u_{j} \Phi_{i}^{\prime}=0, \quad \sum_{j=1}^{p}\left|u_{j}\right|=1 .
$$

(B') Für jedes $j(1 \leqq j \leqq p)$ gelte: Für $\nu \in M_{j}<\{1, \ldots, N\}$ und nur für diese Indizes ist $g_{v}\left(y_{j}\right)=0$. Die Vektoren $g_{v j}^{*}$ sind linear unabhängig, und es gibt reelle Zahlen $v_{v j}\left(v \in M_{j}\right)$, die $\geqq 0$ im Falle $1 \leqq j \leqq r$ und $\leqq 0$ im Falle $r+1 \leqq j \leqq p$ sind, mit

Auf dem linearen Teilraum

$$
\dot{\varphi_{i}}-\dot{\Phi_{j}^{*}}-\sum_{v \in M_{i}} v_{v i} \dot{g_{v i}}=0
$$

$$
H_{j}=\left\{\eta ; \eta^{T} \dot{g}_{v j}=0\left(\nu \in M_{i}\right)\right\} \subset R^{m}
$$

ist die quadratische Form

$$
Q_{j}(\eta)=\eta^{T}\left[\ddot{\varphi_{j}}-\Phi_{j}^{\ddot{1}}-\sum_{\nu \in M_{j}} v_{y_{j}} \ddot{g_{* j}}\right] \eta
$$

negativ definit im Falle $1 \leqq j \leqq r$, positiv definit im Falle $r+1 \leqq j \leqq p$.

(C') Sei $\left\{\eta_{j}^{(k)} ; k=1, \ldots, m-m_{j}\right\}$ eine Basis von $H_{j}(j=1, \ldots, p)$. Durch die linearen Gleichungssysteme

$$
\begin{aligned}
& \eta_{j}^{(k) T}\left[-\Phi_{j}^{\prime} \zeta+\left(\varphi_{j}^{*}-\Phi_{j}^{* *}-\sum_{v \in M_{j}} v_{v j} \dot{g}_{v i}\right) \eta_{j}\right]=0 \\
& \left(k=1, \ldots, m-m_{j} ; j=1, \ldots, p\right)
\end{aligned}
$$

sind jedem $\zeta=\left(\zeta_{1}, \ldots, \zeta_{n-1}\right) \in R^{n-1}$ eindeutig Vektoren $\eta_{1} \in H_{1}, \ldots, \eta_{p} \in H_{p}$ zugeordnet (N.B. das ist keine Voraussetzung, sondern Folgerung aus $\left(\mathrm{B}^{\prime}\right)$ ). Fïr alle $\zeta \in R^{n-1}$ mit

$$
\zeta^{T} \Phi_{1}^{\prime}=\cdots=\zeta^{T} \Phi_{p}^{\prime}=0
$$

und die jeweils zugeordneten $\eta_{1}, \ldots, \eta_{p}$ sei

$$
q\left(\zeta, \eta_{j}\right)=\sum_{j=1}^{p} u_{j}\left\{\zeta^{T} \Phi_{j}^{\prime \prime} \zeta+\eta_{j}^{T}\left(\varphi_{j}-\Phi_{j}^{\ddot{1}}-\sum_{v \in M_{j}} v_{v j} g_{v i}\right) \eta_{j}\right\}<0,
$$

solange nicht $\zeta=0$ und daher $\eta_{1}=\cdots=\eta_{p}=0$ ist.

Sind die Bedingungen $\left(\mathrm{A}^{\prime}\right),\left(\mathrm{B}^{\prime}\right),\left(\mathrm{C}^{\prime}\right)$ erfüllt, so liegt bei $z=\tilde{z}$ ein relatives Minimum der Maximalabweichung

$$
\varrho(z)=\operatorname{Sup}_{y \in \hat{Y}}|\varphi(y)-\Phi(y, z)| \text {. }
$$

Bemerkungen. Der lineare Teilraum $H$ von $\$ 3$ wird eigentlich durch

$$
\zeta^{T} \Phi_{1}^{\prime}=\cdots=\zeta^{T} \Phi_{r}^{\prime}=-\zeta^{T} \Phi_{r+1}^{\prime}=\cdots=-\zeta^{T} \Phi_{p}^{\prime}
$$

beschrieben. Mit (22) ergibt sich jedoch (23). Auf die Formulierung der notwendigen Bedingungen von $\$ 5$ für den Spezialfall der Approximationsaufgabe soll hier verzichtet werden. Jedoch sei bemerkt, daß die lokale Kuhn-TuckerBedingung (7) in diesem Fall mit dem von Meinardus in [5] angegebenen Satz 84 
(§ 8.1) zusammenhängt. Die Aussage, aus der (7) mit dem Lemma von Farkas folgt, besagt dasselbe wie jener Satz; allerdings sind hier nur endlich viele Extremalpunkte zugelassen und es wird eine constraint qualification gefordert. DaB man ohne diese hier nicht auskommt, liegt daran, daB $Y$ nicht als beschränkt vorausgesetzt ist. Im Beispiel mit

$$
\varphi(y)=\frac{1}{y_{1}+1}, \quad \Phi(y, z)=z_{1}, \quad g_{1}(y)=-y_{1},
$$

wo also $\hat{Y}=[0, \infty)$ und $\tilde{z}_{1}=\frac{1}{2}$ mit $\tilde{\varepsilon}=\frac{1}{2}$ die eindeutig bestimmte Extremalstelle ist, gilt jener Satz von Meinardus nicht. Andererseits zeigt der Beweis jenes Satzes, daß bei beschränktem $Y$ im Falle der Approximationsaufgabe die constraint qualification (I) erfüllt ist.

Beispiel. $\varphi(y)=y_{1}$ ist für $0 \leqq y_{1} \leqq 1$ durch $\Phi(y, z)=z_{1}+y_{1}\left(z_{2}+y_{1}\right)^{2}$ zu approximieren. Man findet zwei lokale Minima:

$$
\begin{aligned}
& \tilde{z}=\left(\begin{array}{c}
\sqrt{1 / 27} \\
0
\end{array}\right) \quad \text { mit } \quad \tilde{\varepsilon}=\sqrt{1 / 27} \approx 0,19 \\
& \tilde{z}=\left(\begin{array}{c}
(10-7 \sqrt{7}) / 27 \\
-2
\end{array}\right) \text { mit } \tilde{\varepsilon}=\frac{7 \sqrt{7}-10}{27} \approx 0,34 .
\end{aligned}
$$

In beiden Fällen sind die obigen hinreichenden Bedingungen erfüllt. Mit $\tilde{z}=$ $\left(\frac{1}{2},-1\right)^{T}$ hat man einen weiteren Punkt, an dem die lokale Kuhn-Tucker-Bedingung erfüllt ist, die notwendige Semidefinitheitsbedingung jedoch nicht.

\section{\$9. Verallgemeinerungen}

Die in $\$ 3$ angegebene Aufgabe wurde der Übersichtlichkeit wegen möglichst einfach formuliert. Einige Verallgemeinerungen liegen nahe.

a) Man $\operatorname{kann} Y=Y_{1} \cup \ldots \cup Y_{s}$ wählen, wobei die $Y_{i}$ Teilmengen von Räumen verschiedener Dimension sind. Bei der numerischen Behandlung von Randwertaufgaben monotoner Art nach dem Prinzip der lokal optimalen Schranken [6] kann etwa $Y_{1}$ ein Bereich sein, in dem die Differentialgleichung gefordert ist, $Y_{2}$ sein Rand. Insbesondere können auch isolierte Punkte zu $Y$ gehören oder - anders gesagt - können einzelne Ungleichungen $f_{i}(x) \leqq 0$ hinzutreten. Wie in diesen Fällen die Maximalbedingungen $z u$ formulieren und zu beweisen sind, ist evident.

b) Uberdies dürfen in der Beschreibung von $M$ und $Y$ neben Ungleichungen auch endlich viele Gleichungen vorkommen. Die zugehörigen Multiplikatoren $u_{i}$ bzw. $v_{v j}$ sind dann keinen Vorzeichenbeschränkungen zu unterwerfen.

c) Es ist natürlich nicht wesentlich, daß die auftretenden Funktionen im ganzen Raum definiert sind.

d) Eine naheliegende, aber wohl weder für die Anwendungen noch beweistechnisch interessante Verallgemeinerung der behandelten zweistufigen Aufgaben wären mehrstufige Aufgaben.

e) Im Hinblick auf Anwendungen u.a. in der control theory könnte eine Verallgemeinerung der hier gefundenen Ergebnisse auf abstrakte Räume (z.B. Banachräume statt $R^{n}$ und $R^{m}$ ) wünschenswert sein.

10 Nuner. Math, Bd. 15 


\section{Literatur}

1. Collatz, L.: Approximation in partial differential equations, S. $413-422$ in R. E. Langer (ed.): On numerical approximation. Madison: The University of Wisconsin Press 1959.

2. - Wetterling, W.: Optimierungsaufgaben. Berlin-Göttingen-Heidelberg-New York: Springer 1966.

3. Dieudonné, J.: Foundations of modern analysis. New York-London: Academic Press 1960.

4. McCormick, G. P.: Second order conditions for constrained minima. SIAM J, Appl. Math. 15, 641-652 (1967).

5. Meinardus, G.: Approximation von Funktionen und ihre numerische Behandlung. Berlin-Göttingen-Heidelberg-New York: Springer 1964.

6. Wetterling, W.: Lokal optimale Schranken bei Randwertaufgaben. Computing 3, $125-130$ (1968).

7. - Lösungsschranken bei elliptischen Differentialgleichungen. International Series of Numerical Mathematics 9, 393-401 (1968).

8. - Uber Minimalbedingungen und Newton-Iteration bei nichtlinearen Optimierungsaufgaben. Erscheint in: International Series of Numerical Mathematics. Basel: Birkhäuser.

Prof. Dr. W. Wetterling

Technische Hogeschool Twente

Onderafdeling der Toegepaste Wiskunde

Postbus 217

Enschede/Niederlande 